\title{
Erratum
}

\section{The frequency control of laser diodes}

\author{
M. de Labachelerie, C. Latrasse, P. Kemssu and P. Cerez
}

J. Phys. III France 2 (1992) 1557-1589

I) On the bottom of page 1576 and on the top of page 1577, instead of reading the sentences:

"Some appropriate rotation points have been demonstrated experimentally, $\left(R_{1}[96]\right.$ or $R_{2}$ [99]), however, we have recently determined rigorously the optimal rotation axis position which should be located on the straight line $\left(R_{1} R_{2}\right)$ to suppress mode hopping to the first order, the best point being $R_{4}$ which can provide a theoretical tuning range broader than $30 \mathrm{~nm}$ free of mode hops around $1.5 \mu \mathrm{m}[100] . "$

replace them by:

"Some appropiate rotation points have been demonstrated experimentally, $R_{1}$ [96] or $R_{2}$ [99]. We have recently calculated that the best rotation point is effectively $R_{2}$. This point can provide a theoretical tuning range broader than $30 \mathrm{~nm}$ free of mode hops around $1.5 \mu$ [100]."

II) On the bottom of page 1576, the previous figure 16 and its caption have to be replaced by the following one:

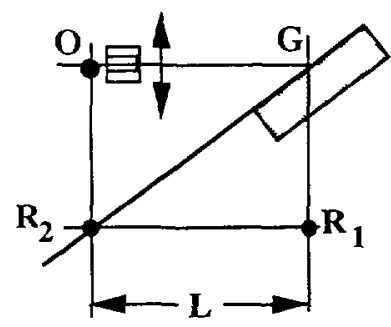

Fig. 16. - Particular rotation axes: $R_{1}$, suggested in [96]; $R_{2}$, suggested in [99]; $R_{2}$ found with our model in [100]. 
